\title{
Du caritatif au politique, l'itinéraire de Jeanne Koehler-Lumière
}

\section{Bernadette ANGLERAUD}

\section{(2) OpenEdition}

1 Journals

Édition électronique

URL : https://journals.openedition.org/clio/4331

DOI : 10.4000/clio.4331

ISSN : 1777-5299

Éditeur

Belin

Édition imprimée

Date de publication : 1 novembre 2006

ISBN : 2-85816-867-9

ISSN : $1252-7017$

Référence électronique

Bernadette ANGLERAUD, « Du caritatif au politique, l'itinéraire de Jeanne Koehler-Lumière », Clio.

Histoire, femmes et sociétés [En ligne], 24 | 2006, mis en ligne le 23 août 2013, consulté le 22 avril 2022. URL : http://journals.openedition.org/clio/4331 ; DOI : https://doi.org/10.4000/clio.4331

Ce document a été généré automatiquement le 22 avril 2022.

Tous droits réservés 


\title{
Du caritatif au politique, l'itinéraire de Jeanne Koehler-Lumière
}

\author{
Bernadette ANGLERAUD
}

1 Comme de nombreuses femmes de son époque, Jeanne Kœhler-Lumière (1870-1926) a un itinéraire personnel éclipsé par la renommée des hommes de sa famille, ses frères Louis et Auguste Lumière, industriels et inventeurs du cinéma. À Lyon, dans le quartier de Monplaisir, fief des établissements Lumière, où le souvenir des célèbres frères est omniprésent - monument commémoratif, avenue des frères Lumière, groupe scolaire Lumière, rue du Premier Film - une rue porte le nom de Jeanne Kœhler, témoignant de son apport original à l'histoire de cette famille d'industriels. Employée à la fabrication des plaques photographiques, Jeanne Kœhler devient dame d'œuvre quand les affaires familiales prospèrent et qu'il s'agit, à l'exemple des femmes de la bourgeoisie lyonnaise, de s'engager dans des actions caritatives en faveur du personnel de l'usine. Comme elles, c'est aussi au chevet des blessés, qu'elle accomplit sa mission durant la guerre. Mais alors que nombre de dames d'œuvre voient leur vocation caritative remise en cause après-guerre par l'implication des pouvoirs publics dans le domaine social, Jeanne Kœhler, au contraire, accompagne la mise en place d'une politique en faveur de l'enfance et des femmes. Présidente de nombreux comités, conférencière, elle devient à Lyon, dans les années 1920, une actrice de premier plan de la politique sociale menée par la municipalité lyonnaise dirigée par le radical Edouard Herriot. L'itinéraire de Jeanne Kœhler, reconstitué à partir des dossiers de décorations honorifiques et d'archives privées, permet ainsi d'appréhender les passerelles qui ont pu exister entre la philanthropie patricienne et la politique sociale dans les années 1920. Passerelles qui ont sans doute joué un rôle majeur dans la réussite du projet.

\section{Dans le sillage des Lumière}

2 L'enfance et la jeunesse de Jeanne Kœhler se déroulent dans l'ombre de l'aventure industrielle des Lumière. L'année de sa naissance, 1870, est aussi celle de l'installation de la famille Lumière à Lyon : Antoine, son père, achète un studio de photographie dans 
le centre ville. En 1881, il acquiert un terrain dans l'Est lyonnais, dans le quartier de Monplaisir, pour y construire une usine de plaques photographiques enduites de l'émulsion au gélatino-bromure d'argent qu'a mise au point son fils, Louis. Dès lors, toute la famille est mobilisée dans l'affaire ; Jeanne, dès l'âge de quinze ans, travaille quotidiennement comme sa mère et ses sœurs, dans l'usine, à la fabrication des fameuses plaques à «Étiquette bleue $»^{1}$. Elle partage le quotidien des ouvrières au sein d'une entreprise familiale qui débute modestement et difficilement. C'est seulement à partir des années 1890 que vient la prospérité. Jeanne s'éloigne des affaires Lumière en épousant, à l'âge de 20 ans, René Kohler, originaire des Vosges, docteur en sciences naturelles et en médecine à $23 \mathrm{ans}^{2}$. Sa nomination en 1889 à la Faculté de Lyon lui fait rencontrer la fille d'industriels lyonnais qui bénéficient déjà d'une certaine renommée sur la scène locale. Pour autant, Jeanne Kœhler ne rompt pas avec le clan familial. Le jeune couple s'installe à proximité de l'usine et des villas Lumière et René Kœhler est associé aux affaires, comme membre du conseil d'administration des Usines Lumière, puis administrateur délégué de la Société des Produits chimiques créée par les Lumière en 1902 à laquelle il apporte ses connaissances scientifiques. C'est également grâce à la collaboration de son beau-frère qu'Auguste Lumière peut se lancer dans le domaine médical ${ }^{3}$. Les affaires se développant et se diversifiant, René Kœehler apporte la caution scientifique et surtout médicale qui manquait aux frères Lumière.

Jeanne Kœhler, quant à elle, se mobilise dans l'action sociale qui se développe autour de l'entreprise, comme la plupart des femmes et filles d'entrepreneurs chargées d'assister les familles du personnel. De nombreuses œuvres lyonnaises participent à cet élan caritatif ${ }^{4}$. Mais les orientations idéologiques des Lumière éloignent Jeanne Kœhler de ces sociétés qui, pour la plupart, lient action caritative et évangélisation. Dans la bataille entre Église et État républicain, particulièrement animée à Lyon, la famille Lumière se situe clairement dans le camp républicain et laïque. Antoine Lumière est franc-maçon et affiche ouvertement son anti-cléricalisme, ce qui l'isole de la bourgeoisie traditionnelle lyonnaise. René Kœhler est également maçon même s'il est moins actif que son beau-père. L'engagement social des Lumière s'ancre donc dans le camp laïque, proche des actions menées par les municipalités radicales, particulièrement celle de Herriot à partir de 1905.

\section{Le soutien à l'école laïque}

Assez tôt, se mettent en place au sein des usines Lumière des structures d'assistance : caisses de retraite, services médicaux, aide aux femmes enceintes (la main-d'œuvre étant majoritairement féminine). Comme les établissements industriels sont installés au coeur d'un quartier populaire qui leur fournit la main-d'œuvre nécessaire, la politique sociale de l'entreprise se prolonge par une participation active des Lumière à la vie locale. Les industriels aident financièrement chorales et autres associations de quartier, mais c'est surtout autour de l'école publique que se cristallise leur action. Dès 1880 à Lyon, la municipalité a laïcisé les écoles primaires publiques congréganistes tandis que les catholiques organisaient des souscriptions et créaient des écoles libres. Après le vote des lois scolaires, le conflit s'est aiguisé entre les deux camps et l'enseignement est devenu un des terrains privilégiés d'affrontement entre partisans de l'ordre ancien et défenseurs de l'État républicain. Ainsi, la Ligue des femmes françaises (LFF) créée en 1901 à Lyon, sous le patronage de l'archevêque ${ }^{5}$, fait signer des pétitions 
et quête pour les écoles libres et le soutien des candidats catholiques aux élections. Dans le même temps, l'activité anticléricale des loges maçonniques s'intensifie.

5 Tandis que s'organise la concurrence entre écoles libres et laïques, l'enjeu du conflit porte aussi sur les activités extrascolaires, une manière d'investir le temps libre des enfants. Dans le camp laïque, on tente de parachever la laïcisation de l'enseignement en créant tout un réseau de cours pour adolescents, de mutualités scolaires, de patronages $^{6}$. Les femmes sont appelées à concurrencer les dames patronnesses catholiques en devenant animatrices d'œuvres d'éducation populaire. La Ligue de l'Enseignement, créée par Jean Macé en 1866 pour défendre la laïcité, accorde à partir de 1894 une grande importance à ces activités. Dans chacun de ses cercles, elle met en place un " comité de Dames " pour gérer et développer les associations de loisirs et de culture populaire. Sa stratégie est très proche de celle des œuvres caritatives puisqu'elle fait appel à des femmes qui ont «fortune, loisirs, peuvent et doivent se vouer pour combattre le bon combat $! »^{7}$. La famille Lumière est de ce combat. Antoine Lumière adhère totalement au projet scolaire de la Troisième République. Pour cet autodidacte, l'éducation est un droit pour tous, quel que soit le milieu social ou le sexe. Le fait de scolariser ses enfants, filles et garçons, et d'envoyer ses fils aînés à la Martinière - une école professionnelle qui associe enseignement général et pratique en atelier ${ }^{8}$ - témoigne de l'importance qu'Antoine Lumière accordait à un enseignement qui lui avait fait défaut. C'est à l'échelle locale, en soutenant l'école publique la plus proche des usines, que le clan Lumière œuvre concrètement en faveur de la laïcité. Dès leur arrivée dans le quartier de Monplaisir, les Lumière adhèrent à la Société d'Encouragement aux écoles laïques créée en 1881 ; à partir de 1890 c'est un gendre d'Antoine, le Docteur Armand Gélibert, qui en devient président; elle prend alors le nom de Denier des Écoles et organise des souscriptions pour financer l'équipement des écoles et les repas des enfants. Le Denier fédère tous les membres de la famille Lumière, les fils et gendres d'Antoine Lumière se retrouvant dans le conseil d'administration et René Kœhler à la présidence de la société.

6 Jeanne Kœhler, elle aussi, s'engage dans cette action. Elle a une trentaine d'années et c'est aussi au Denier des Écoles de Monplaisir qu'elle fait ses premières armes. Elle y crée l'œuvre du Vestiaire puis un Comité des Dames pour développer les activités extrascolaires, conformément aux directives de la Ligue de l'Enseignement. La « section féminine du Denier », joue un rôle important dans la sociabilité du milieu patronal local. Nombre d'épouses d'industriels s'y retrouvent pour collecter vêtements, livres et jeux au profit des enfants, mais aussi réfléchir aux initiatives à mettre en oeuvre. Parmi elles, on peut citer Pauline Lafont, épouse d'Adolphe Lafont, qui est à la tête d'une entreprise de confection, spécialisée dans le vêtement de travail et située dans le voisinage des usines Lumière. Les filles et belles-filles d'Antoine Lumière sont également mobilisées dans ce comité dont Jeanne Kœhler assure la présidence. Mais celle-ci ne se contente pas de cette action caritative en harmonie avec les choix doctrinaux des hommes de la famille. Sa première expérience auprès des enfants d'âge scolaire la conduit à s'intéresser au sort des plus jeunes, pour qui se pose un problème de garde dans un quartier où les femmes sont nombreuses à travailler à l'usine. Ses préoccupations croisent celles des pouvoirs publics qui cherchent à développer, tant au niveau national que local, une politique en faveur de la petite enfance ${ }^{9}$. Dans les années 1900, la lutte contre la mortalité infantile et l'anticléricalisme se conjuguent pour inciter le camp républicain à prendre en charge les enfants en bas âge. La protection 
des nourrissons impose de proposer des solutions alternatives à l'abandon ou à la mise en nourrice en favorisant les crèches et salles d'asile qui, en 1900, relèvent encore majoritairement d'initiatives privées $^{10}$. À Lyon, sous les municipalités du radical Gailleton puis du socialiste Augagneur, l'enjeu consiste à implanter dans les quartiers des salles d'asile, devenues écoles maternelles et crèches, pour concurrencer les structures privées d'obédience catholique. Cette politique est l'occasion, pour Jeanne Kœhler, de concrétiser son intérêt pour la petite enfance. Ainsi, collabore-t-elle activement à l'Cuuvre des petits de la maternelle du VII e arrondissement qui supervise la mise en place d'un réseau de salles d'asile. Elle obtient la mise à disposition, par la ville de Lyon, d'une propriété dans les environs, à Brignais, pour y envoyer les jeunes enfants en séjour à la campagne.

7 Dans les années 1890-1900, de nombreux rapports de l'inspection générale de l'enfance mettent en évidence la précarité, l'insuffisance et le caractère privé des crèches dans le Rhône ${ }^{11}$. S'appuyant sur ce constat, le maire, Victor Augagneur, lance en 1901 un projet de construction de crèches et de réhabilitation des établissements appartenant à la Société protectrice de l'Enfance. Il s'agit de compléter le dispositif républicain initié par les lois Ferry sur l'école primaire; "la municipalité prendra ainsi l'enfant à sa naissance et sous les yeux de ses parents, le fera passer successivement dans les différentes stations de son système d'instruction et d'éducation et le conduira de cette façon à l'âge où il pourra affronter les exigences de la vie ${ }^{12}$ ». C'est à Jeanne Koehler que revient la charge de veiller au bon fonctionnement de la crèche municipale qui ouvre le $1^{\text {er }}$ janvier 1912 dans le quartier de Monplaisir. Comme les autres structures, cet établissement doit assumer une triple mission de garde des nourrissons, de suivi médical par le biais des consultations régulièrement pratiquées par un médecin et de formation en puériculture pour les mères, laquelle est assurée par Jeanne Kohler qui vient « encourager les mamans à se perfectionner dans l'art de soigner leurs petits et leur distribuer des prix pour créer entre elles une bienfaisante émulation ${ }^{13}{ }$. C'est bien dans le sillage du paternalisme d'entreprise, à l'échelle du quartier, que cette dame d'œuvre laïque s'est initiée à l'action sociale. Cette première expérience lui permet de s'insérer dans un réseau de sociabilité qui regroupe les milieux de la bourgeoisie républicaine et laïque. Son activisme sur le terrain lui vaut également de se faire remarquer des pouvoirs municipaux qui sont à la recherche de relais locaux. Ainsi, dès les années 1900, elle est chargée de faire appliquer, dans son quartier de Monplaisir, les directives municipales en matière d'aide à l'enfance. La guerre va constituer un tournant dans son engagement social. Comme beaucoup d'autres femmes de son milieu, elle vit le conflit dans les hôpitaux au chevet des soldats blessés ${ }^{14}$. Mais ses engagements antérieurs et l'activisme du clan Lumière lui valent d'assumer des responsabilités qui dépassent le simple service d'infirmière.

\section{Les œuvres de guerre}

8 Sa première expérience a lieu à Cherbourg dès septembre 1914. Alors âgée de 44 ans, Jeanne Kœhler suit sa fille, épouse depuis quelques mois d'un officier de marine muté à Cherbourg. Du 15 septembre 1914 au 15 avril 1915, elle est infirmière bénévole à l'Hôpital des contagieux installé au Casino de Cherbourg. Sa participation à l'Union des Femmes Françaises, une des sociétés de la Croix-Rouge ${ }^{15}$, lui avait permis dès avant 1914 de se préparer à cette activité de soignante. Au printemps 1915, elle est de retour à 
Lyon pour œuvrer dans l'hôpital auxiliaire organisé à Monplaisir par son frère Louis Lumière. Elle y travaille comme infirmière aux côtés de ses sœurs et belles-sœurs, tandis que son époux, René, et son beau-frère, Armand Gélibert, y participent en qualité de médecins. La même année, Jeanne Kœhler va donner une nouvelle dimension à sa mission de soignante, en acceptant de seconder son frère Auguste à l'Hôtel-Dieu (le grand hôpital de Lyon). Depuis 1896, ce dernier, profitant de la prospérité de l'entreprise Lumière et de la présence à Lyon d'une école médicale de pointe, a créé un laboratoire de physiologie expérimentale où il travaille avec plusieurs personnalités du corps professoral des facultés des sciences et de médecine. En 1910, le laboratoire est transféré dans un local plus vaste et complété par une clinique où les produits, après avoir été élaborés et validés, sont mis à disposition des malades. Ainsi, de 1899 à 1914 sont mises au point plusieurs médications, commercialisées par une société spécifique : la Société des Produits Chimiques Spéciaux, dont la présidence du conseil d'administration est assurée par Auguste Lumière et René Kœhler. L'importance prise par la société conduit le professeur Léon Bérard, chirurgien des hôpitaux et membre de l'académie de médecine, à solliciter la collaboration d'Auguste Lumière dans son service hospitalier de l'Hôtel-Dieu. En 1914, il obtient l'affectation de l'industriel au service de santé. Ensemble, ils travaillent sur le traitement des blessés atteints par le tétanos. Auguste Lumière est également en charge du service radiologique de l'HôtelDieu, mobilisant l'usine de Monplaisir pour développer les clichés. L'industriel entraîne dans son sillage sa fille Andrée et sa sœur Jeanne. Cette dernière partage ses journées entre le service radiologique, où elle seconde son frère, et les salles d'opération où elle assiste le professeur Léon Bérard. Dès la période de guerre, elle est distinguée par les autorités publiques, recevant en 1916 la médaille d'argent des épidémies, puis l'insigne or des infirmières en 1918.

9 L'expérience de la guerre a donné une nouvelle dimension à l'engagement social de Jeanne Kœhler. Son réseau relationnel s'étend désormais bien au-delà du quartier de Monplaisir tandis que son action dépasse la simple assistance aux familles qui gravitent autour des usines Lumière. Le côtoiement des souffrances et des horreurs de la guerre renforce son militantisme, tandis qu'elle acquiert des compétences professionnelles aux côtés de son frère et de médecins. Après le conflit, un des ses premiers engagements concerne l'aide aux victimes de la guerre, prolongeant ainsi l'action qu'elle a menée durant quatre ans. C'est au sein de l'Union des Femmes Françaises, liée à la Croix-Rouge ${ }^{16}$ (Jeanne Kohler fait désormais partie du conseil d'administration) qu'elle réalise son projet. L'objectif est en effet de venir en aide aux victimes civiles et militaires du conflit, en prenant par exemple en charge des maisons de convalescence pour les blessés et l'aide aux rapatriés. Conciliant cette mission et son engagement au service de l'enfance, Jeanne Kœhler participe, dès le jour de sa fondation au printemps 1918, à l'Office départemental des pupilles de la nation. Membre élu de cet office, elle siège dans la commission qui statue sur les subventions à apporter aux familles d'orphelins pour leur entretien, leur instruction, leur santé et leur établissement. Pour autant, elle n'abandonne pas l'action sur le terrain, frappant aux portes pour collecter les fonds nécessaires. Ainsi, en 1920, quand Louis Lumière organise une exposition pour présenter au public son invention de la photographie en relief, elle obtient de lui que le fruit de la vente des premiers portraits revienne intégralement aux pupilles de la nation ${ }^{17}$. 


\section{Du caritatif au politique}

10 Après la guerre, Jeanne Kœhler ploie sous les décorations officielles qui viennent récompenser son dévouement auprès des blessés mais aussi officialiser son action au profit de l'enfance. L'année 1920 la voit gratifiée de la médaille de la reconnaissance française tandis que les hospices civils de Lyon lui remettent la médaille de l'administration de l'Hôtel-Dieu. En 1921, ce sont les palmes d'officier d'académie, puis en 1925 vient la consécration avec la croix de chevalier de la légion d'honneur. Ces distinctions, exceptionnelles à l'époque pour une femme, récompensent des actions et des engagements dans des secteurs qui sont alors au cœur des préoccupations de l'État. À partir des années 1920, l'aura de Jeanne Kœhler est utilisée par les pouvoirs publics qui en font la propagatrice d'une nouvelle politique sociale, marquée à Lyon, comme à l'échelle nationale, par des préoccupations natalistes jointes à un interventionnisme d'État ${ }^{18}$. L'un des volets majeurs de cette politique est la protection infantile. Après l'hécatombe de la guerre, la lutte contre la dépopulation est érigée en priorité nationale ${ }^{19}$ et relayée par les pouvoirs locaux. "Il nous faut lutter contre la dépopulation, défendre les mères et les enfants, améliorer le logement ouvrier, combattre l'alcoolisme et la tuberculose ", écrit Edouard Herriot aux lendemains du conflit $^{20}$, esquissant ainsi les axes d'une ambitieuse politique sociale dont les objectifs fondamentaux sont l'encouragement de la natalité et le recul de la mortalité, notamment infantile. La " défense des mères et des enfants » aboutit à la mise en place de structures qui s'appuient sur la nouvelle législation de protection des femmes enceintes (loi Strauss de 1913) et des nouveaux-nés (loi de 1919 sur les primes d'allaitement). "Le problème de l'enfance lyonnaise est un de ceux qui m'ont le plus préoccupé. J'ai tenté de multiplier les œuvres à l'usage des tout petits ", déclare en 1921 le maire de Lyon ${ }^{21}$. Mais pour mettre en œuvre cette politique, la municipalité a besoin d'acteurs expérimentés en attendant que n'émerge un corps de professionnelles du social $^{22}$. Le milieu caritatif peut constituer un vivier, mais à Lyon la dominante catholique et conservatrice $d u$ monde des dames d'œuvre exclut toute idée de collaboration avec la municipalité radicale d'Edouard Herriot. C'est donc dans l'aile laïque et républicaine qu'il lui faut chercher des volontaires. Expérimentée, auréolée de sa notoriété, Jeanne Kœhler est la candidate idéale qui, non seulement va mettre en œuvre la politique municipale, mais s'en faire aussi l'ambassadrice.

11 Un des premiers domaines d'action concerne les maisons maternelles, dont l'objectif est d'accueillir des femmes de milieux défavorisées (souvent célibataires), avant et après l'accouchement, afin qu'elles n'abandonnent pas leur enfant ${ }^{23}$. Avant la guerre, quelques institutions privées se chargeaient de cette mission dans une démarche explicite de "réhabilitation morale " de ces jeunes femmes. C'est le cas, à Lyon, de l'œuvre de la Samaritaine, fondée en 1891 à Monplaisir (le quartier des Lumière), qui reçoit des femmes enceintes primipares "victimes d'une première faute", pour les accueillir ensuite avec leur nouveau-né24. Après la guerre de 1914-1918, l'initiative est reprise par les pouvoirs publics dont le premier objectif est la lutte contre la mortalité infantile. Les maisons maternelles proposent aux femmes enceintes sans ressource, mariées ou non, un asile afin de veiller au bon déroulement de la grossesse puis à la santé des nouveaux-nés. Le but poursuivi n'est plus moral mais bien démographique. Il s'agit de préserver des enfants mis en danger par la situation précaire de leur mère. À Lyon, deux initiatives répondent à cet objectif. En 1918, Edouard Herriot inaugure, dans 
la périphérie méridionale de Lyon, la maison des mères, au château de Gerland ${ }^{25}$ : un " asile» de 35 lits pour recevoir les femmes enceintes sans domicile qui sont ensuite accueillies avec leur bébé après leur accouchement à l'hôpital de la charité. En 1920, une structure similaire est fondée à Bron, autre commune au sud-est de l'agglomération lyonnaise. Il s'agit de la nourricerie départementale du Vinatier ${ }^{26}$, qui reçoit, à leur sortie de la maternité, des femmes sans ressources avec leur nourrisson. L'originalité de ce dernier établissement tient au fait que chaque mère accueillie s'engage à nourrir également un nourrisson pupille de l'assistance publique. Ces maisons s'insèrent dans le dispositif de lutte contre la mortalité infantile en agissant contre les abandons d'enfants et pour la promotion de l'allaitement maternel. Ces initiatives sont complétées à Lyon, comme dans d'autres villes, par l'ouverture de restaurants où les mères nourrices peuvent venir se restaurer gratuitement, par la construction de crèches municipales et par une politique active en matière de suivi médical des nourrissons.

Jeanne Kœhler est d'emblée associée à ces actions. Aux côtés d'élus du conseil général et de médecins, elle est vice-présidente de la commission de contrôle de la nourricerie $\mathrm{du}$ Vinatier. Aux fonctions administratives, elle ajoute des visites régulières aux pensionnaires qu'elle entend réconforter et conseiller. Son action est déterminante dans la réussite du projet: dès la première année, 507 femmes et 48 pupilles sont accueillis. Elle est également chargée de superviser le devenir des femmes et enfants après leur séjour de trois à six mois dans ces établissements et elle préside, à ce titre, le Comité de patronage des nourrissons et des mères à leur sortie des maisons maternelles. Sa tâche est complexe car elle doit faire le lien avec les visiteuses de l'enfance ${ }^{27}$, nouvellement mises en place et chargées du suivi médical, avec les crèches qui accueillent les enfants, mais aussi avec les services administratifs pour assurer aux jeunes femmes le paiement des allocations auxquelles elles ont droit. Son expérience, tant dans le domaine de la petite enfance que dans le secteur médical, lui permet d'assurer ce rôle en coordonnant les actions des différents acteurs.

Dans les années 1920, Jeanne Kœhler est ainsi devenue un personnage important dans le domaine de l'action sociale à Lyon. En témoignent ses très nombreux titres et fonctions : membre du conseil d'administration de l'office départemental des pupilles de la nation du Rhône, vice-présidente de la section cantonale du $7^{\mathrm{e}}$ arrondissement des pupilles de la vallée du Rhône, présidente du Comité de patronage des mères et des nourrissons, vice-présidente de l'œEuvre pour les petits de la maternelle et de la commission de contrôle de la Nourricerie du Vinatier. Jeanne Kœhler se voit également confier par la municipalité la mission de devenir une "propagatrice» de la campagne nataliste engagée. À cette fin, elle est nommée présidente de l'Organisation de la section lyonnaise d'hygiène, qu'elle représente au conseil national. Plusieurs rapports lui sont confiés sur la lutte contre la mortalité infantile ou sur la protection de la mère et de l'enfant du premier âge. Des années 1924 à 1926, elle anime de nombreuses conférences sur ces thèmes auprès de publics variés - mères des maisons maternelles, visiteuses de l'enfance, élèves infirmières ou encore membres du conseil municipal de Lyon. Aux côtés du docteur Vigne, directeur du bureau municipal d'hygiène de la ville de Lyon et ami des Lumière, Jeanne Kohler devient en quelque sorte un des lieutenants de la politique municipale, mais aussi nationale, dans la lutte contre la mortalité infantile et la mise en place d'une politique de l'enfance. 

Légion d'honneur, Jeanne Kœhler fait l'objet d'un hommage unanime auquel participent toutes les grandes personnalités de la ville ${ }^{28}$. Dès les années suivantes, les initiatives se multiplient afin de pérenniser son souvenir. Une fondation Jeanne Kœhler destinée aux enfants des écoles est créée par le Denier de Monplaisir, première association caritative à laquelle elle avait participé, tandis que la ville de Lyon donne, dès 1927, son nom à une rue du quartier de Monplaisir.

L'itinéraire de Jeanne Kœhler l'a conduite des œuvres caritatives à une collaboration active avec les pouvoirs publics pour la mise en place d'une politique sociale municipale dans les années 1920. Le point de départ de son engagement est en partie lié à la réussite des affaires familiales, puisqu'elle quitte l'atelier pour devenir dame d'œuvre aux côtés de ses belles-sœurs, Rose et Marguerite Lumière. Comme pour nombre de jeunes femmes de la bourgeoisie, la guerre est un tournant; à la fois un traumatisme par le contact brutal avec les réalités les plus dures et la fin d'une époque puisqu'à la charité succède l'assistance publique. C'est dans ce contexte que Jeanne Kœhler commence une carrière dans le champ social mais aussi politique. Sœur d'industriels, épouse d'un médecin universitaire, elle s'engage aux côtés des pouvoirs publics dans l'action en faveur de l'enfance, apportant l'aura de la famille Lumière qui est parvenue, dans les années 1920, à conquérir la scène lyonnaise par sa réussite économique, par le dévouement et le talent reconnus à Auguste Lumière sur le plan médical, mais aussi par son soutien fidèle à Edouard Herriot. L'expérience de Jeanne Kœhler et sa notabilité sont des atouts pour des pouvoirs publics à la recherche d'acteurs compétents, capables d'impulser une politique sociale encore balbutiante ${ }^{29}$. Le savoir-faire de Jeanne Kœhler, qui ne se départira jamais des méthodes des dames d'œuvre - jouant sur les relations interpersonnelles et sur la confiance «de femme à femme »- permet de passer à la mise en œuvre concrète d'objectifs généraux. La lutte contre la dépopulation et pour l'amélioration de la santé publique, en particulier du côté des enfants a été lancée par des politiques, des économistes et des médecins mais elle ne peut porter ses fruits que grâce à des relais gagnés à cette cause. Jeanne Kohler est un de ces relais, capable de convaincre aussi bien les mères des quartiers populaires que les élites bourgeoises auxquelles elle appartient. Sur la scène lyonnaise, elle a eu avec quelques autres femmes - dont Léonie Gillet, épouse de l'industriel Edmond Gillet, à la tête du groupe chimique du même nom-, un rôle de "passeuse" du caritatif au social dans cette période charnière des années 1920-1930.

Jeanne Kœhler-Lumière a incarné, par son engagement, une forme originale de participation des femmes à la vie politique, à une époque où celles-ci n'étaient pas citoyennes. Une situation, à première vue paradoxale, qui ne semble pourtant guère avoir préoccupé cette « entrepreneuse » du social. 


\section{BIBLIOGRAPHIE}

ANGLERAUD Bernadette et PELLISSIER Catherine, 2003, Les dynasties lyonnaises, des Morin-Pons aux Mérieux du XIX ${ }^{\mathrm{e}}$ siècle à nos jours, Paris, Perrin.

AUDET Fabrice, 1997, « L'école de la Martinière. Les élèves des promotions 1900-1909 », Bulletin du Centre Pierre Léon, $n^{\circ}$ 3-4.

BEC Colette, 1994, Assistance et République : la recherche d'un nouveau contrat social sous la III ${ }^{\mathrm{e}}$ République, Paris, L'Atelier.

COVA Anne, 2000, Au service de l'Église, de la patrie et de la famille : femmes catholiques et maternité sous la III ${ }^{\mathrm{e}}$ République, Paris, L'Harmattan.

DESSERTINE Dominique et MARADAN Bernard, 2002, « Patronages catholiques, patronages laïques », Cahiers d'histoire, tome $47, \mathrm{n}^{\circ} 1 / 2$.

Dossier des Archives municipales nº 9, 1995, Lumière l'album de famille, Lyon.

DUBESSET Mathilde et ZANCARINI-FOURNEL Michelle, 1993, Parcours de femmes, réalités et représentations, Saint-Étienne 1880-1950, Lyon, PUL.

DUMONS Bruno, 2006, Les dames de la Ligue des femmes françaises (1901-1914), Paris, Le Cerf.

FAYET-SCRIBE Sylvie, 1990, Associations féminines et catholicisme, de la charité à l'action sociale $\mathrm{XIX}^{\mathrm{e}}-\mathrm{XX}^{\mathrm{e}}$, Paris, éditions ouvrières.

HERRIOT Edouard, 1952, Jadis, d'une guerre à l'autre (1914-1936), Paris, Flammarion.

HERVIER Marcel, 1921, Les oeuvres de l'enfance à Lyon, Lyon.

KNIBIEHLER Yvonne, 1980, Nous, les assistantes sociales. Naissance d'une profession (1930-1960), Paris Aubier.

LUMIERE Auguste, 1953, Mes travaux et mes jours, Paris, La Colombe.

NOAILLY Denys, 1927, Les maisons maternelles, la Nourricerie départementale du Vinatier, thèse de médecine, Lyon.

PELLISSIER Catherine, 1996, Loisirs et sociabilités des notables lyonnais au XIX $\mathrm{e}^{\mathrm{e}}$ siècle, Lyon,

ELAH.

PERRET Emmanuel, 1919, Les visiteuses de l'enfance, le District Nursing. Un essai d'hygiène social par la Croix-Rouge américaine à Lyon, thèse de médecine, Lyon.

PETIT Edouard, 1902, l'Ecole de demain : autour de l'école, œuvres vues, des fêtes, de l'école au lycée, femmes et hommes d'œuvre, vers l'éducation sociale, Paris, Picard.

ROLLAND Frédéric, 1928, Les maisons maternelles, la Maison des Mères de Gerland de la Ville de Lyon, thèse de médecine, Lyon.

ROLLET Catherine, 1990, La politique à l'égard de la petite enfance sous la III ${ }^{e}$ République, Paris, INED.

SMITH Bonnie, 1981, Les bourgeoises du Nord, Paris, Perrin.

THEBAUD Françoise, 1986, La femme au temps de la guerre de 14, Paris, Stock. 
THEBAUD Françoise, 1985, « Le mouvement nataliste dans la France de l'Entre-deux-guerres, l'Alliance nationale pour l'accroissement de la population française ", Revue d'Histoire Moderne et Contemporaine, t. 33.

THEBAUD Françoise, 1986, Quand nos grands-mères donnaient la vie : la maternité en France dans l'entre-deux-guerres, Lyon, PUL.

VACHET, 1900, Lyon et ses œuvres, Lyon, E. Vitte.

\section{NOTES}

1. Lumière 1953 : 29.

2. Notice René Kœhler, Lumière l'album de famille, dossier Archives municipales nº 9, Lyon, 1995 : 43-44.

3. Un autre gendre d'Antoine Lumière joue un rôle majeur, le Dr Armand Gélibert qui l'assistera aussi dans ses expérimentations médicales.

4. Nombreux sont alors les répertoires qui énumèrent ces œuvres, cf. Sabran 1893 ; Vachet 1900.

5. Fayet-Scribe 1990 ; Dumons 2006.

6. Dessertine et Maradan 2002.

7. Petit 1902.

8. L'école professionnelle de la Martinière, créée par la ville de Lyon en 1826, devint, dans la deuxième moitié du XIX siècle, une pépinière d'entrepreneurs. Cf. Audet 1997.

9. Rollet 1990.

10. Cova 2000.

11. Archives départementales du Rhône, Série 3X Assistance publique, dossier 3X 1852.

12. Archives départementales du Rhône, Série 3X Assistance publique, dossier 3X 1853.

13. Passage de l'allocution du Professeur Bertin, le 5 décembre 1926 en hommage à la mémoire de Madame Kœhler. Un texte révélateur du regard porté par les élites très préoccupées d'hygiène et de santé publique (dont beaucoup de femmes) sur ces mères qu'il faut «éduquer » en particulier les mères des classes populaires.

14. Thébaud 1992.

15. Cette UFF n'a rien à voir avec l'organisation du même nom créée à l'automne 1944, dans la mouvance du Parti communiste.

16. Archives départementales du Rhône, Série $4 \mathrm{M}$ Associations, dossier $4 \mathrm{M} 528$.

17. Ce qui rapporte 35000 francs, cf. notice Jeanne Kœhler, Lumière l'album de famille, dossier Archives municipales n 9, Lyon, $1995:$ 55-56.

18. Bec 1994.

19. Thébaud 1985.

20. Herriot 1952.

21. Préface d'Edouard Herriot au livre de Hervier 1921.

22. C'est en 1932 qu'est institué le diplôme d'État d'assistante sociale; la loi Fié rendant obligatoire, un an auparavant, l'obtention d'un diplôme pour l'exercice de professions médicosociales. Cf. Knibiehler 1980.

23. Sur les maisons maternelles, cf. Thébaud 1986; Dubesset et Zancarini-Fournel 1993, 3 ème partie, Maternités.

24. Archives départementales du Rhône, dossier 216 W 194.

25. Rolland 1928.

26. Noailly 1927.

27. Perret 1919. 
28. Le Progrès, 27 novembre 1926 : « devant l'église Saint-Maurice, autour du char funéraire, se reconnaissaient la plupart des notabilités de Lyon: M. le préfet Vallette, M. le premier adjoint Emmanuel Lévy, M. le recteur Gheust, M. Depéret, doyen de la faculté des sciences, M. Victor Grignard directeur de l'école de chimie, M. le docteur Michon, président du syndicat des médecins"

29. Diebolt 2001.

\section{RÉSUMÉS}

L'itinéraire de Jeanne Kœhler-Lumière l'a conduite de la philanthropie à la collaboration avec les pouvoirs publics pour la réalisation d'une politique sociale à Lyon après la Première Guerre mondiale. Fille et sœur d'industriels, elle participe aux œuvres mises en place pour le personnel de l'usine familiale, puis élargit son action en faveur de l'enfance à l'échelle de la ville. Cependant, l'ancrage de la famille Lumière dans le camp de la République laïque isole Jeanne Kœhler-Lumière des milieux traditionnels de la philanthropie conservatrice et catholique. La guerre constitue un tournant durant lequel elle s'engage dans les services de santé, travaillant avec des sommités du monde médical lyonnais. Son expérience et sa notabilité lui valent dans les années 1920 d'être sollicitée par la municipalité radicale en quête de compétences pour impulser une politique sociale. Dame d'œuvres puis ambassadrice du social, Jeanne Kœhler-Lumière illustre une forme originale de participation des femmes à la vie politique.

Jeanne Kœhler-Lumière's journey took her from philanthropy to collaboration with public authorities as she contributed to the elaboration of social policy after World War I. As the daughter and sister of industrialists, she participated in the charities set up for the personnel at the family factory and then extended her work on behalf of children to the municipal level. However, the Lumière family's strong identification with the secular republicans isolated Jeanne Kœhler-Lumière from the traditional conservative and Catholic philanthropic circles. The war constituted a turning point during which she became involved in health services, working with the leading experts of Lyon's medical community. Her experience and her respectability became valuable in the 1920s when she was called upon by the radical city council looking for skilled individuals to establish new social policies. Jeanne Kœhler-Lumière's itinerary from a Lady of charity to an ambassadress for social services illustrates a new kind of participation of women in political life.

\section{INDEX}

Mots-clés : action sociale, assistance, enfance, industrie, municipalité, œuvres, paternalisme, philanthropie

Index géographique : Lyon 


\section{AUTEUR}

\section{BERNADETTE ANGLERAUD}

Bernadette ANGLERAUD, ancienne élève de l'École Normale Supérieure, agrégée, docteur en histoire, enseigne à l'Université Grenoble 1 et est membre associée du LARHRA (Laboratoire de Recherche Historique Rhône-Alpes). Après avoir travaillé sur la petite bourgeoisie (Les boulangers lyonnais aux XIX ${ }^{e}-X X^{e}$ siècles, 1998, Paris, éditions Christian), ses recherches se sont orientées sur les bourgeoisies industrielles à travers le cas lyonnais. Elle a publié notamment en collaboration avec Catherine Pellissier, 2003, Les dynasties lyonnaises des Morin-Pons aux Mérieux, du XIXe à nos jours, Paris, Perrin ; et en collaboration avec Catherine Pellissier, Jean Etèvenaux, Guetty Long, Lyonnaises d'hier et d'aujourd'hui, 2005, Lyon, éditions Bellier (chapitres économie et philanthropie). 